\title{
Systemic Lupus Erythematosus and Angioedema: A Cross-Sectional Study From the National Inpatient Sample
}

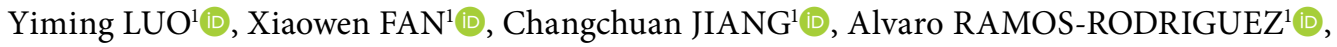

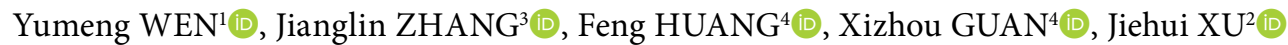 \\ ${ }^{1}$ Department of Medicine, Mount Sinai St. Luke's and Mount Sinai West Hospitals, New York, United States \\ ${ }^{2}$ Department of Medicine, Weill Cornell Medical College, New York, United States \\ ${ }^{3}$ Department of Rheumatology and Immunology, China Pla General Hospital, Beijing, China \\ ${ }^{4}$ Department of Respiratory Medicine, China Pla General Hospital, Beijing, China
}

\begin{abstract}
Objectives: This cross-sectional study aims to investigate the odds of developing angioedema (AE) in systemic lupus erythematosus (SLE) populations compared to non-SLE populations in hospital settings in the United States using a nationwide database.

Materials and methods: We used the data from the National Inpatient Sample for the years 2012 to 2014. We constructed two models for multivariate logistic regression analysis. Model 1 was designed to adjust demographic information, while model 2 included each factor in model 1 and additionally accounted for AE-related comorbidities.

Results: A total of 90,485 hospitalizations with an AE diagnosis were identified for the years 2012 to 2014, among which 1,505 hospitalizations had both SLE and AE. Compared to those without SLE, AE patients with SLE were younger and included more females. In AE hospitalizations, SLE was associated with higher odds of AE-related comorbidities including atopic disorder, leukocytoclastic vasculitis, eosinophilia, and infections. SLE was associated with higher odds of $A E$ both as all inpatient diagnosis and as principal diagnosis (unadjusted odds ratio [OR] $3.24,95 \%$ confidence interval [Cl] 2.87-3.63, $\mathrm{p}<0.001$, model 1 adjusted OR 2.54, 95\% Cl 2.26-2.86, $\mathrm{p}<0.001$, model 2 adjusted OR $1.71,95 \% \mathrm{Cl} 1.51-1.93, \mathrm{p}<0.001)$.

Conclusion: Our study demonstrates that SLE is associated with higher odds of having AE, including severe $A E$ as the principal reason for inpatient admission. SLE is possibly an independent risk factor for AE.

Keywords: Angioedema; cross-sectional study; epidemiology; National Inpatient Sample; systemic lupus erythematosus.
\end{abstract}

Angioedema (AE) is transient localized subcutaneous and mucosal non-pitting edema due to temporary increase in vascular permeability caused by the release of vasoactive mediators. ${ }^{1}$ Though self-limiting in nature, AE may present with life-threatening airway swelling which requires hospitalization. $\mathrm{AE}$ may be divided into allergic or non-allergic AE. Non-allergic $\mathrm{AE}$ may be further subdivided into hereditary angioedema (HAE), acquired $\mathrm{AE}$ with $\mathrm{C} 1$ inhibitor deficiency (C1-INH-AAE, previously referred to as acquired $\mathrm{AE}$ ), renin-angiotensin-aldosterone system blocker-induced $\mathrm{AE}$, pseudoallergic $\mathrm{AE}$, or idiopathic $\mathrm{AE} .{ }^{1}$ Rare causes of $\mathrm{AE}$, including those associated with hypereosinophilic syndrome and hypocomplementemic urticarial vasculitis, have been described in the literature as well. ${ }^{2,3}$

Systemic lupus erythematosus (SLE) is a prototypical autoimmune disease that affects

Received: August 18, 2018 Accepted: November 20, 2018 Published online: January 28, 2019

Correspondence: Yiming Luo, MD. Department of Medicine, Mount Sinai St. Luke's and Mount Sinai West Hospitals, 10019 New York, United States. Tel: 1-917-480-9748 e-mail:yiming.luo@mountsinai.org

\section{Citation:}

Luo Y, Fan X, Jiang C, Ramos-Rodriguez A, Wen Y, Zhang J, et al. Systemic lupus erythematosus and angioedema: A cross-sectional study from the national inpatient sample. Arch Rheumatol 2019;34(3):301-307. 
more than 300,000 people in the United States (US) and millions worldwide. ${ }^{4}$ SLE is characterized by multi-system involvement, autoantibody formation, and dysregulation of the complement system. Previous case reports and case series studies have identified an uncommon association between SLE and two rare types of AE, HAE. 5,6 and C1-INH-AAE. ${ }^{7-10}$ Nonetheless, epidemiological studies of the two immune system-related conditions are lacking. Therefore, in this crosssectional study, we aimed to investigate the odds of developing $\mathrm{AE}$ in SLE populations compared to non-SLE populations in hospital settings in the US using a nationwide database.

\section{MATERIALS AND METHODS}

This study was conducted at St. Luke's and Mount Sinai West Hospitals between November 2017 and July 2018. We used data from the National Inpatient Sample (NIS) for the years 2012 to 2014 . The NIS is the largest publicly available inpatient database in the US, representing a $20 \%$ stratified sample of all US non-federal hospitals, and is sponsored by the Agency for Healthcare Research and Quality and the Healthcare Cost and Utilization Project (HCUP).11,12 After weighing, the data reflects over $95 \%$ of all hospitalizations within the US, which totals to approximately 35 million each year. The NIS contains data elements from inpatient discharge records, including demographic, disposition, diagnostic, and procedural information, while lacking detailed clinical course, laboratory, and pharmacy data. Diagnoses were identified using the International Classification of Diseases Ninth Revision (ICD-9) codes. This study did not require approval from the Institutional Review Board at Icahn School of Medicine at Mount Sinai because no identifiable private information was obtained and/or available from the NIS database. The study was conducted in accordance with the principles of the Declaration of Helsinki.

Healthcare Cost and Utilization Project data quality report for the NIS database is publicly available for each year. Reports for the years 2012 to 2014 were reviewed and missing data rates were detected that were consistently lower than $0.5 \%$ for most data elements. Our approach to address those with missing data rates above $0.5 \%$ was described below in the statistical analysis section.

We included hospital encounters for patients with a primary or secondary diagnosis of $\mathrm{AE}$ (ICD-9 code 995.1) from years 2012 to 2014. It should be noted that each sample in NIS represents an encounter for hospitalization, not an individual patient. SLE was identified using ICD-9 code 710.0. ICD 9 codes used to identify comorbidities related to AE were listed in Table 1.

Our primary outcome was the odds ratio (OR) of hospitalizations with a diagnosis of $\mathrm{AE}$ and a concurrent diagnosis of SLE compared to those without SLE. We also compared the demographic information, AE-related comorbidities, and systemic comorbidities in AE patients with or without SLE.

In the US, Medicare and Medicaid are government-sponsored medical insurance plans which primarily cover vulnerable populations, including elderly or low-income individuals as well as those with permanent disabilities. ${ }^{13}$ Medical insurance status was used in our study as a surrogate marker for patients' socioeconomic status (SES). ${ }^{14}$ In addition, median household income for patients' postal code was also used as another marker for patients' SES.

\section{Statistical analysis}

To represent the national hospitalization data, analyses accounted for the complex survey design and stratification and clustering of the data per NIS database sets. Student's t-tests were performed for continuous variables and chi-squared analysis was performed for categorical variables. We constructed two models for multivariate logistic regression analysis. Model 1 was designed to adjust demographic information, including age, sex, race/ethnicity, and primary payer. Model 2 included each factor in model 1 and additionally accounted for AE-related comorbidities, which includes atopic disorder (allergic rhinitis, atopic dermatitis, and asthma), leukocytoclastic vasculitis (including urticarial vasculitis), medication adverse effects, eosinophilia, infections, anaphylaxis, congenital enzyme deficiencies (including $\mathrm{C} 1-\mathrm{INH}$ deficiency), and lymphoproliferative disease. Sensitivity analysis was performed by using AE as the principal diagnosis for hospitalization 
Table 1. International Classification of Diseases Ninth Revision code used to identify diagnosis

\begin{tabular}{ll}
\hline Angioedema & 995.1 \\
Systemic lupus erythematosus & 710 \\
Atopic disorder (allergic rhinitis, atopic dermatitis and asthma) & $477 . x, 691 . x, 693 . x x$ \\
Leukocytoclastic vasculitis (including urticarial vasculitis) & 446.29 \\
Eosinophilia & 288.3 \\
Infections & $001-137,460-466,480-488,590 . x, 595.0,599.0,955.91$, \\
& 785.52 \\
Anaphylaxis & $995.6 x, 999.4 x, 995.0$ \\
Congenital enzyme deficiencies (including C1-INH deficiency) & 277.6 \\
Lymphoproliferative disease & $200 . x x, 201 . x x, 202 . x x, 203 . x x, 204 . x x, 273.1,238.6,238.72$, \\
& $238.73,238.74,238.75,238.77$ \\
Hypertension & $401 . x, 405 . x x$ \\
Diabetes mellitus & $250 . x x$ \\
Coronary artery disease & $410-414$ \\
Congestive heart failure & $428 . x x$ \\
Chronic obstructive pulmonary disease & $491 . x x, 492 . x$ \\
Chronic kidney disease or glomerulopathy & $580 . x x, 585 . x$ \\
Chronic liver disease & $571 . x x, 070 . x x$ (excluding 070.0, 070.01, 070.20, 070.21, 070.30, \\
& $070.31,070.41,070.43,070.51,070.53)$ \\
\hline C1-INH: C1 inhibitor. & \\
\hline
\end{tabular}

instead of both primary and secondary diagnoses, an approach which included the most clinically significant $\mathrm{AE}$ that led directly to hospitalization. The Stata software (StataCorp LLC, Texas, USA) was used for statistical analyses.

Of the variables used in our study, only race had a missing data rate above $0.5 \%$. We assumed that data was not missing at random and applied the multivariate imputation by chained equations method. ${ }^{15}$ The multiple imputations procedure was based on a multinomial logistic regression model with five iterations that included the rest of the variables. The results for the primary and secondary outcomes were compared before and after accounting for missing data and were found to be similar. Therefore, the results were presented without accounting for the missing data.

\section{RESULTS}

A total of 90,485 hospitalizations with a diagnosis of $\mathrm{AE}$ were identified between years 2012 to 2014 , among which 1,505 hospitalizations were found to have concurrent diagnoses of both SLE and AE. The characteristics of AE patients with or without SLE were shown in Table 2. Demographically, compared to those without SLE, AE patients hospitalized with SLE were younger (mean age 44 vs. 58 years, $p<0.001$ ), more likely to be female ( $89 \%$ vs. $57 \%, p<0.001$ ), more likely to be African-American (57\% vs. 38\%, $\mathrm{p}<0.0001$ ), more likely to have Medicaid as their medical insurance ( $25 \%$ vs. $16 \%, p<0.001)$, and more likely to be from the lowest income quartile areas ( $45 \%$ vs. $36 \%, p=0.002 \%)$.

For comorbidities related to $\mathrm{AE}$, those with SLE were associated with a higher likelihood of atopic disorders (allergic rhinitis, atopic dermatitis, and asthma, $18 \%$ vs. $13 \%$, $\mathrm{p}=0.008$ ), leukocytoclastic vasculitis (including urticarial vasculitis, $0.33 \%$ vs. $0.04 \%, p=0.029$ ), eosinophilia ( $1 \%$ vs. $0.24 \%, p=0.010)$, and infections $(40 \%$ vs. $24 \%, p<0.001)$, but not with medication adverse effects, anaphylaxis, congenital enzyme deficiencies (including C1-INH deficiency), or lymphoproliferative disease. For category of infections, AE with SLE was associated with a numerically higher likelihood of upper respiratory infection, pneumonia or influenza, urinary tract infection, cellulitis or erysipelas and sepsis, but was statistically significant only 
Table 2. Demographic and clinical features in angioedema hospitalizations with or without systemic lupus erythematosus

\begin{tabular}{|c|c|}
\hline AE with SLE $(n=1,505)$ & AE without SLE $(n=88,980)$ \\
\hline $\mathrm{n}$ & $\%$ \\
\hline
\end{tabular}

\section{Demographic information}

Mean age (year)

44

58

$<0.001$

Sex

Female

\section{Race/Ethnicity}

Caucasian

African-American

23

57

Hispanic

Asian or other

\section{Medical insurance status}

Medicare

Medicaid

Private insurance

Other

Median household income for patient's postal code

First (lowest) quartile

Second quartile

Third quartile

Fourth (highest) quartile

\section{AE-related comorbidities}

Atopic disorder

Anaphylaxis

Medication adverse effects

Leukocytoclastic vasculitis (including urticarial vasculitis)

Eosinophilia

Congenital enzyme deficiencies (including C1-INH deficiency)

Lymphoproliferative disease

Infections (total)

Upper respiratory infection

Pneumonia or influenza

Urinary tract infection

Cellulitis or erysipelas

Sepsis

\section{Systemic comorbidities}

Hypertension

Diabetes mellitus

Coronary artery disease

Congestive heart failure

Chronic obstructive pulmonary disease

Chronic kidney disease or glomerulopathy

Chronic liver disease

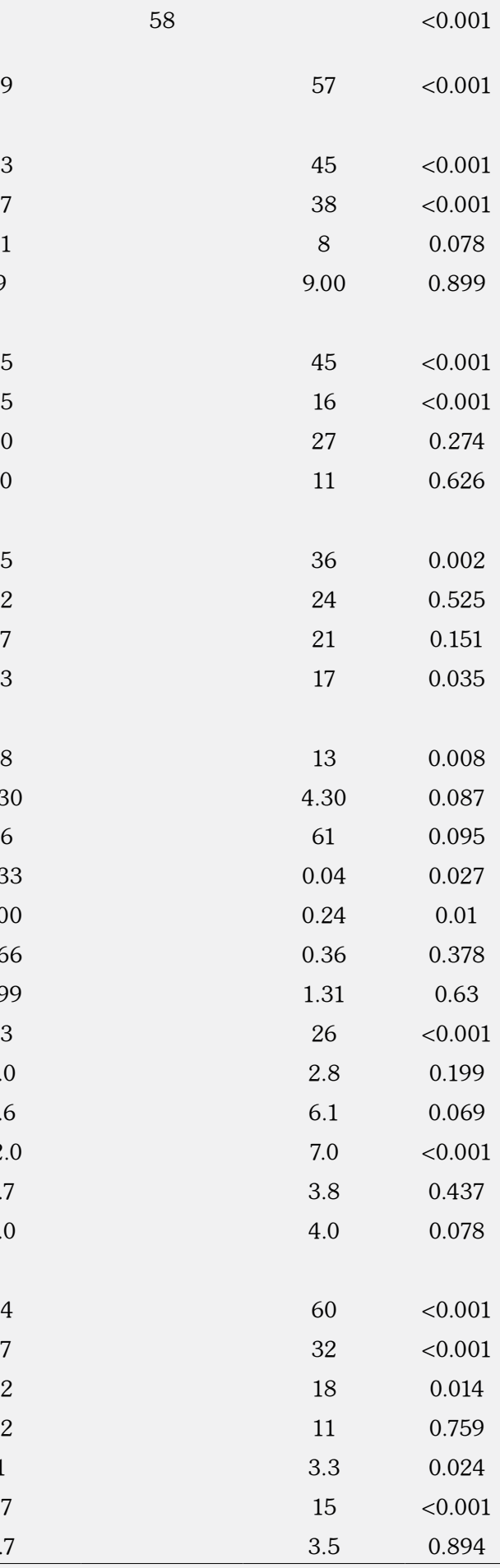

AE: Angioedema; SLE: Systemic lupus erythematosus; C1-INH: C1 inhibitor. 
Table 3. Odds of angioedema in those with or without systemic lupus erythematosus

\begin{tabular}{|c|c|c|c|c|c|c|c|c|c|}
\hline & \multicolumn{3}{|c|}{ Univariable logistic regression } & \multicolumn{3}{|c|}{$\begin{array}{l}\text { Multivariable logistic regression } \\
\text { (Model 1) }\end{array}$} & \multicolumn{3}{|c|}{$\begin{array}{l}\text { Multivariable logistic regression } \\
\text { (Model 2) }\end{array}$} \\
\hline & OR & $95 \% \mathrm{CI}$ & $p$ & OR & $95 \% \mathrm{CI}$ & $p$ & OR & $95 \% \mathrm{CI}$ & $p$ \\
\hline AE all inpatient diagnoses & 3.24 & $2.87-3.63$ & $<0.001$ & 2.54 & $2.26-2.86$ & $<0.001$ & 1.71 & $1.51-1.93$ & $<0.001$ \\
\hline AE principal diagnoses & 2.50 & $2.12-2.96$ & $<0.001$ & 1.86 & $1.56-2.22$ & $<0.001$ & 1.25 & $1.04-1.49$ & 0.018 \\
\hline
\end{tabular}

for higher likelihood of urinary tract infection (12.0\% vs. $7.0 \%, \mathrm{p}<0.001)$ (Table 2 ).

For systemic comorbidities, AE patients with SLE were associated with more chronic kidney disease/glomerulopathy ( $37 \%$ vs. $15 \%, \mathrm{p}<0.001$ ) but less hypertension ( $44 \%$ vs. $60 \%, p<0.001)$, diabetes mellitus $(17 \%$ vs. $32 \%, \quad p<0.001)$, coronary artery disease ( $12 \%$ vs. $18 \%, p=0.014)$, and chronic obstructive pulmonary disease ( $1 \%$ vs. $3.3 \%, p=0.024)$. There was no statistical significance between SLE and congestive heart failure and chronic liver disease in $\mathrm{AE}$ patients (Table 2).

Compared to those without SLE, patients with SLE were at increased odds of having a diagnosis of AE (unadjusted OR: 3.24 95\% confidence interval [CI] (2.87-3.63), $\mathrm{p}<0.001$; model 1 adjusted OR: $2.5495 \%$ CI (2.26-2.86), $\mathrm{p}<0.001$; model 2 adjusted OR: 1.71 95\% CI (1.51-1.93), $\mathrm{p}<0.001)$ in the hospital. Sensitivity analysis was performed using $\mathrm{AE}$ as primary diagnosis for hospitalization instead of both primary and secondary diagnoses, which was consistent with our finding that SLE was associated with increased odds of $\mathrm{AE}$ (unadjusted OR: 2.50 95\% CI (2.12-2.96), $\mathrm{p}<0.0001$. Model 1 adjusted OR: 1.86, 95\% CI (1.56-2.22), $\mathrm{p}<0.0001$. Model 2 adjusted OR: 1.25 95\% CI (1.04-1.49), $\mathrm{p}=0.018$ ) (Table 3).

\section{DISCUSSION}

To the best of our knowledge, this is the first epidemiological study on the association between SLE and AE. Our study showed that compared with those without SLE, AE patients with SLE were younger and more likely to be females and African-Americans, which is consistent with the epidemiological features of those with SLE. ${ }^{4}$ For AE-related comorbidities, those with SLE were more likely to have an atopic disorder, leukocytoclastic vasculitis, eosinophilia, and infections; while not likely to have anaphylaxis, medication adverse effects, congenital enzyme deficiencies or lymphoproliferative disease.

Our analysis showed that those with SLE were at higher risk of having a diagnosis of $\mathrm{AE}$ in the hospital and that it may be an independent risk factor after adjusting for the above known risk factors. In addition, SLE was also associated with the most clinically significant $\mathrm{AE}$ which led directly to hospitalization in our sensitivity analysis. $\mathrm{AE}$ is a potentially life-threatening condition which may be caused by different underlying etiology and pathophysiology and categorized as mast cell mediated or bradykinin-mediated mechanism, or those of unknown mechanism.

Angioedema is most commonly mediated by mast cell degranulation, which is often associated with urticaria and may be triggered by allergic reactions and medications. ${ }^{1}$ The association between SLE and allergic diseases is under debate and previous studies have yielded conflicting results. ${ }^{16-18}$ In our study, we found higher odds of atopic disorders among AE patients with SLE, implying that higher risk of allergic reaction may contribute to more AE in this population. SLE has also been linked to chronic spontaneous urticaria, which is also mast cell mediated and may manifest with AE. ${ }^{19}$

Systemic lupus erythematosus has also been associated with rare types of $\mathrm{AE}$ mediated by C1-INH deficiency or dysfunction, which includes HAE and C1-INH-AAE. For HAE, Donaldson et al. ${ }^{5}$ identified three out of 220 HAE patients with lupus-like disorders but with only cutaneous involvement. Similarly, Brickman et al. ${ }^{6}$ found one SLE case and one drug-induced lupus case 
among 157 patients with HAE. Based on these findings, the estimated prevalence of lupus in patients with $\mathrm{HAE}$ is $2 \%$, but the prevalence of SLE in AE would be expected to be lower than $1 \%$. The pathophysiology was proposed to be linked to the modification of complement metabolism, leading to accumulation of immune complexes and decreased viral clearance. ${ }^{20}$ In our study, however, we failed to detect statistically significant differences for congenital enzyme deficiencies among AE patients with or without SLE. This may due to a combination of the rarity of $\mathrm{HAE}$, the rare association between the two conditions, and the limitation of ICD-9 codes, which cannot differentiate hereditary C1-INH deficiency from other congenital enzyme deficiencies.

The association between C1-INH-AAE and SLE is more complicated and not without controversies. C1-INH-AAE is a rare condition with only several hundred cases reported in the literature. ${ }^{21}$ C1-INH-AAE has been traditionally categorized into two subtypes: (i) one with increased catabolism of $\mathrm{C} 1-\mathrm{INH}$ and associated with lymphoproliferative disorder, and (ii) one associated with autoantibodies to C1-INH. ${ }^{21}$ However, there were case reports of C1-INH-AAE associated with SLE which did not fit into either two categories. Cacoub et al. ${ }^{9}$ described a "third type" of C1-INH-AAE in clinically quiescent SLE patients with transit low C1-INH antigenic and functional levels and hypocomplementemia, both of which normalized with resolution of $\mathrm{AE}$ after immunosuppressive therapy. Tekin et al. ${ }^{10}$ recently summarized $18 \mathrm{AAE}$ cases associated with SLE. He demonstrated that the diagnosis of SLE and AE was concomitantly established for eight patients. ${ }^{10}$ Seventeen patients were with low complement levels, five patients presented with glomerulonephritis, and three patients developed neuropsychiatric lupus. ${ }^{10}$ Intriguingly, SLE patients found to have either low C1-INH ${ }^{22}$ levels or high $\mathrm{C} 1-\mathrm{INH}$ autoantibodies ${ }^{23}$ did not necessarily develop clinical AE.

There are several limitations of our study. First, bias from unmeasured confounders may exist given our retrospective cross-sectional design. Second, there was a risk of misclassification bias while using ICD-9 codes, and it was also unknown how often $\mathrm{AE}$ was coded as a diagnosis if the $\mathrm{AE}$ was merely a clinical manifestation of a systemic disorder, such as anaphylaxis. ICD-9 codes in secondary diagnosis may also have not been able to distinguish acute in-hospital events from comorbidities. In addition, since NIS is an inpatient database, conditions commonly encountered in outpatient settings may not always be accurately documented, such as atopic disorders or urticaria. Furthermore, due to the nature of our database, the results only represent hospitalized populations and may not be generalizable to other settings. Last but not least, as described above, $\mathrm{AE}$ is a highly heterogeneous condition and we were not able to further categorize different types of $\mathrm{AE}$.

In conclusion, our study is the first populationbased cross-sectional study on the association between SLE and AE in hospitalized population. Our findings show that those with SLE are at higher odds of having $\mathrm{AE}$ in inpatient setting, including severe $\mathrm{AE}$ as the principal reason for inpatient admission after adjusting for major comorbidities and medication adverse effects. Our study highlights that SLE is possibly an independent risk factor for AE. Thus, clinicians should be vigilant for $\mathrm{AE}$ when managing patients with SLE in the hospital. Prospective studies are required to confirm the relationship between SLE and AE, while further investigations are also warranted to elucidate the types and clinical features of AE in SLE.

\section{Declaration of conflicting interests}

The authors declared no conflicts of interest with respect to the authorship and/or publication of this article.

\section{Funding}

The authors received no financial support for the research and/or authorship of this article.

\section{REFERENCES}

1. Lewis LM. Angioedema: etiology, pathophysiology, current and emerging therapies. J Emerg Med 2013;45:789-96.

2. Weller PF, Bubley GJ. The idiopathic hypereosinophilic syndrome. Blood 1994;83:2759-79.

3. Saigal K, Valencia IC, Cohen J, Kerdel FA. Hypocomplementemic urticarial vasculitis with angioedema, a rare presentation of systemic lupus erythematosus: rapid response to rituximab. $\mathrm{J} A \mathrm{Am}$ Acad Dermatol 2003;49:283-5. 
4. Carter EE, Barr SG, Clarke AE. The global burden of SLE: prevalence, health disparities and socioeconomic impact. Nat Rev Rheumatol 2016;12:605-20.

5. Donaldson VH, Hess EV, McAdams AJ. Lupuserythematosus-like disease in three unrelated women with hereditary angioneurotic edema. Ann Intern Med 1977;86:312-3.

6. Brickman CM, Tsokos GC, Balow JE, Lawley TJ, Santaella M, Hammer $\mathrm{CH}$, et al. Immunoregulatory disorders associated with hereditary angioedema. I. Clinical manifestations of autoimmune disease. J Allergy Clin Immunol 1986;77:749-57.

7. Lahiri M, Lim AY. Angioedema and systemic lupus erythematosus--a complementary association? Ann Acad Med Singapore 2007;36:142-5.

8. Thong BY, Thumboo J, Howe HS, Feng PH. Life-threatening angioedema in systemic lupus erythematosus. Lupus 2001;10:304-8.

9. Cacoub P, Frémeaux-Bacchi V, De Lacroix I, Guillien F, Kahn MF, Kazatchkine MD, et al. A new type of acquired $\mathrm{C} 1$ inhibitor deficiency associated with systemic lupus erythematosus. Arthritis Rheum 2001;44:1836-40.

10. Tekin ZE, Yener GO, Yüksel S. Acquired angioedema in juvenile systemic lupus erythematosus: case-based review. Rheumatol Int 2018;38:1577-84.

11. Databases H. Healthcare Cost and Utilization Project - Overview of the National (Nationwide) Inpatient Sample (NIS) Agency for Healthcare Research and Quality 2016 Available from: www.hcup-us.ahrq.gov/ nisoverview.jsp.

12. Steiner C, Elixhauser A, Schnaier J. The healthcare cost and utilization project: an overview. Eff Clin Pract 2002;5:143-51.

13. Altman D, Frist WH. Medicare and Medicaid at 50 Years: Perspectives of Beneficiaries, Health Care Professionals and Institutions, and Policy Makers. JAMA 2015;314:384-95.
14. Sule S, Petri M. Socioeconomic status in systemic lupus erythematosus. Lupus 2006;15:720-3.

15. Azur MJ, Stuart EA, Frangakis C, Leaf PJ. Multiple imputation by chained equations: what is it and how does it work? Int $\mathrm{J}$ Methods Psychiatr Res 2011;20:40-9.

16. Hsiao YP, Tsai JD, Muo $\mathrm{CH}$, Tsai $\mathrm{CH}$, Sung FC, Liao YT, et al. Atopic diseases and systemic lupus erythematosus: an epidemiological study of the risks and correlations. Int $\mathrm{J}$ Environ Res Public Health 2014;11:8112-22.

17. Sekigawa I, Yoshiike T, Iida N, Hashimoto H, Ogawa $H$. Allergic diseases in systemic lupus erythematosus: prevalence and immunological considerations. Clin Exp Rheumatol 2003;21:117-21.

18. de Lucena GR, Gonçalves RT, Braz AS, Freire EA. Systemic lupus erythematosus and allergy: an integrative review of the literature. Acta Reumatol Port 2013;38:162-70.

19. Kolkhir P, Pogorelov D, Olisova O, Maurer M. Comorbidity and pathogenic links of chronic spontaneous urticaria and systemic lupus erythematosus--a systematic review. Clin Exp Allergy 2016;46: 275-87.

20. Gallais Sérézal I, Bouillet L, Dhôte R, Gayet S, Jeandel PY, Blanchard-Delaunay C, et al. Hereditary angioedema and lupus: A French retrospective study and literature review. Autoimmun Rev 2015;14:564-8.

21. Otani IM, Banerji A. Acquired C1 Inhibitor Deficiency. Immunol Allergy Clin North Am 2017;37:497-511.

22. Nakamura S, Yoshinari M, Saku Y, Hirakawa $\mathrm{K}$, Miishima C, Murai $\mathrm{K}$, et al. Acquired C1 inhibitor deficiency associated with systemic lupus erythematosus affecting the central nervous system. Ann Rheum Dis 1991;50:713-6.

23. Mészáros T, Füst $G$, Farkas $H$, Jakab L, Temesszentandrási G, Nagy G, et al. C1-inhibitor autoantibodies in SLE. Lupus 2010;19:634-8. 